\title{
Hispanismes
}

Revue de la Société des Hispanistes Français

\section{Sociolingüística y comunicación en el marco del contacto vasco-castellano}

Sociolinguistique et communication dans le contexte du contact basque-espagnol Sociolinguistics and communication in the context of Basque-Spanish contact

\section{Sara Gómez Seibane}

\section{(2) OpenEdition}

\section{Journals}

\section{Edición electrónica}

URL: https://journals.openedition.org/hispanismes/771

DOI: 10.4000/hispanismes.771

ISSN: 2270-0765

Editor

Société des Hispanistes Français

Referencia electrónica

Sara Gómez Seibane, «Sociolingüística y comunicación en el marco del contacto vasco-castellano», HispanismeS [En línea], 16 | 2020, Publicado el 01 diciembre 2020, consultado el 01 septiembre 2021. URL: http://journals.openedition.org/hispanismes/771 ; DOI: https://doi.org/10.4000/hispanismes.771

Este documento fue generado automáticamente el 1 septiembre 2021.

es contenus de cette revue sont mis à disposition selon les termes de la Licence Creative Commons Attribution - Pas d'Utilisation Commerciale - Pas de Modification 4.0 International. 


\title{
Sociolingüística y comunicación en el marco del contacto vasco- castellano
}

Sociolinguistique et communication dans le contexte du contact basque-espagnol Sociolinguistics and communication in the context of Basque-Spanish contact

\author{
Sara Gómez Seibane
}

\section{NOTA DEL AUTOR}

Investigación realizada en el proyecto de investigación de excelencia «COREC. Corpus oral de referencia del español en contacto. Fase I: lenguas minoritarias» dirigido por A. Palacios (UAM) y S. Gómez Seibane (UR), referencia/AEI/ PID2019/105865GB-I00.

Agradezco encarecidamente a los dos revisores anónimos los cambios y sugerencias que han contribuido a mejorar este trabajo.

\section{Introducción}

1 Los escenarios de lenguas en contacto son multidisciplinares y, por ello, abordables desde diferentes perspectivas, agrupables fundamentalmente en torno a tres áreas: el individuo bilingüe, la comunidad de habla bilingüe y el sistema lingüístico. Los resultados del contacto lingüístico son igualmente diversos y variados, dependientes a su vez de los diferentes factores que modulan la situación del contacto. Sin embargo, estamos familiarizados con análisis relativamente aislados de los fenómenos de contacto lingüístico. En efecto, en la práctica académica son comunes los enfoques lingüísticos sobre los tipos de cambios que se producen, sus características estructurales y los principios y procesos que implican; los enfoques sociolingüísticos, centrados en la propagación del cambio por medio de las redes que vinculan a los individuos entre sí; y los enfoques psicolingüísticos sobre el modo en que los sistemas 
lingüísticos interactúan en la mente del individuo. A pesar de este procedimiento, las diferentes visiones de la interacción entre lenguas y sus resultados se encuentran estrechamente conectados. Ahora bien, conseguir una explicación interconectada de los diversos aspectos del contacto lingüístico es una tarea extremadamente difícil ${ }^{1}$.

2 En los estudios sobre este asunto, cada vez hay más pruebas de que gran parte del comportamiento lingüístico surge en la interacción de los hablantes, cuyas estrategias están condicionadas por factores sociales, así como por las limitaciones de procesamiento lingüístico y las características de las lenguas implicadas. Por ello, los objetivos de este trabajo son, en primer lugar, ofrecer un resumen del contexto sociolingüístico del contacto vasco-castellano ${ }^{2}$ en las últimas décadas y, en segundo lugar, enmarcado en el contexto sociolingüístico descrito, exponer dos resultados de tipo lingüístico e interactivo-comunicativo. Ello permitirá, por un lado, avanzar en el conocimiento de este escenario de tanto interés para los estudios de contacto $\mathrm{y}$, por otro lado, trazar algunas vías futuras de investigación que pasan por una mejor comprensión de los factores lingüísticos, sociolingüísticos y psicolingüísticos que actúan en estos escenarios.

3 Para ello, este trabajo se estructura en cinco apartados, contando con este primero de tipo introductorio. En el segundo, se aborda la situación histórica de contacto de castellano y lengua vasca, y se resumen los resultados de las políticas lingüísticas de las últimas décadas del siglo XX en lo que respecta al conocimiento de la lengua vasca. En el tercer apartado, se exponen las actitudes de los hablantes hacia la lengua minorizada, tanto las de los bilingües ${ }^{3}$ como las de los monolingües en castellano. A continuación, el apartado cuarto se centra en la comunicación en la situación de contacto, a partir de dos posibles resultados del mismo, como son los cambios inducidos por contacto (4.1), en castellano y en lengua vasca, así como el cambio de código en la conversación (4.2). Como se reúne en los apartados tercero y cuarto información basada en diferentes trabajos, en cada uno de ellos se precisa oportunamente el perfil sociolingüístico del hablante sobre el que se han obtenido los datos. El trabajo se cierra con una recapitulación de los aspectos más importantes y de las líneas de investigación más apremiantes.

\section{Evaluación sociolingüística de la situación de contacto vasco-castellano}

4 La convivencia de la lengua vasca con las lenguas romances se remonta a la romanización y se hace más intensa desde finales de la Edad Media. Con los inicios de la Edad Moderna, el avance románico, y más concretamente el de las hablas castellanas, se acelera sobre todo en los núcleos urbanos (Bilbao, Vitoria y Pamplona), que paulatinamente se convierten en zonas bilingües $\mathrm{y}$, a la vez, en centros de irradiación de castellanización. Lo anterior supone, por un lado, una paulatina pérdida de hablantes de la lengua vasca desde el siglo XVI, que se intensifica durante el siglo XIX por la alfabetización en castellano; y, por otro lado, el refuerzo de la situación de diglosia entre el castellano, considerada la lengua de cultura y del poder económico y social, y la lengua vasca, identificada con la lengua oral de la masa popular ${ }^{4}$.

5 A la vista del progresivo desplazamiento lingüístico del vasco, desde mediados del siglo XIX, las elites económicas y culturales vascas inician actividades en favor del euskera y 
sus tradiciones culturales para protegerlas y recuperarlas, iniciativas que hacia finales del siglo XIX se vincularon a movimientos e ideologías nacionalistas 5 . A su vez, a lo largo del último cuarto del siglo XIX y gran parte del XX, el castellano se convierte en lengua nacional y única en la educación y el gobierno, con políticas autoritarias y abiertamente represivas sobre el uso público de la lengua vasca en varios momentos históricos, como las dictaduras de Primo de Rivera (1923-1931) y Franco (1939-1975). Con la democracia en 1978, el uso de la lengua vasca es un derecho garantizado legalmente por el Estatuto de Autonomía del País Vasco (1979) en su Ley 10/1982 de normalización del uso de la lengua vasca (llamada Ley del Euskera), y que rige para el territorio de la Comunidad Autónoma Vasca (CAV), formado por las provincias de Álava / Araba, Bizkaia y Gipuzkoa. Se trata de un modelo de planificación lingüística cuyo objetivo es garantizar la continuidad y el uso social del euskera tanto en la administración como en la educación, y en un marco de convivencia con el uso del castellano. En cuanto a la Comunidad Foral de Navarra (CFN), en su Ley Foral de 1986 adoptó una política de zonificación que divide su espacio en zonas (vascófona, mixta y no vascófona), con la aplicación de diferentes políticas lingüísticas en cada una de ellas. Así, en las dos primeras zonas se usa el euskera en la educación pública, pero en la tercera, no, por lo que la oficialidad del euskera queda limitada a una parte del territorio ${ }^{6}$.

6 Actualmente, según datos de 2011, en la CAV, de las 2.056.136 personas censadas, el $36,4 \%$ es bilingüe (definido como los que hablan bien o bastante bien) en castellano y lengua vasca, el 19,3\% es bilingüe pasivo en lengua vasca (con competencias sobre todo receptivas), mientras que el $44,3 \%$ restante es monolingüe mayoritariamente en castellano $^{7}$. Desde la aplicación de políticas lingüísticas en la CAV puede observarse un periodo de expansión funcional de la lengua vasca, con un creciente número de hablantes bilingües con distinto grado de dominancia de esta. Efectivamente, desde 1981 hasta 2011, el porcentaje de bilingües ha crecido un 14,5\% (del 21,9\% al 36,4\%). Asimismo, los bilingües pasivos han aumentado su presencia, del 12,2\% de la población al $19,3 \%$. Por el contrario, los monolingües en castellano han descendido de forma notable en estos 30 años: 21,6 puntos menos (del 65,9\% al 44,3\%). Por otro lado, no todos los territorios de la CAV presentan la misma difusión del bilingüismo. Según datos de 2011, la población alavesa bilingüe asciende al 22,9\%, en Bizkaia alcanza el 30,3\%, mientras que en Gipuzkoa representa algo más de la mitad de la población (52,6\%).

Tabla 1. Porcentaje de la competencia lingüística en la CAV (1981-2011)8.

\begin{tabular}{|l|l|l|l|l|}
\hline & 1981 & 1991 & 2001 & 2011 \\
\hline bilingües & $21,9 \%$ & $26,4 \%$ & $32,2 \%$ & $36,4 \%$ \\
\hline bilingües pasivos en euskera & $12,2 \%$ & $16,2 \%$ & $18,2 \%$ & $19,3 \%$ \\
\hline monolingües en castellano & $65,9 \%$ & $57,4 \%$ & $49,6 \%$ & $44,3 \%$ \\
\hline
\end{tabular}

7 Con respecto a los datos de la CFN sobre conocimiento de la lengua vasca ${ }^{9}$, de la población de 16 años o más en 2018, el 14,1\% son vascohablantes, es decir, hablan bien o bastante bien en euskera; los vascohablantes receptores (con algún conocimiento de euskera pero que no llegan a hablarlo bien), el 8\%; y los no vascohablantes representan 
la mayoría de la población, el 77,9\%. Como puede comprobarse, el conocimiento de la lengua vasca es sensiblemente menor en la CFN que en la CAV y, simultáneamente, los datos sobre su evolución en los últimos 40 años también arrojan un ligero aumento del número de hablantes: en 1991, los vascohablantes son el 9,5\%; en 2001, el 10,3\% y en 2011, el 11,7\%. Desde 1991 hasta 2018, el porcentaje de bilingües ha crecido en 4,6 puntos porcentuales (frente a los 14,5 puntos de la CAV entre 1981 y 2011).

En definitiva, la situación descrita es un interesante escenario para los estudios de contacto lingüístico. Por un lado, gracias a las políticas lingüísticas de las últimas décadas del siglo XX, hay más hablantes con competencias lingüísticas en lengua vasca en áreas muy pobladas de la CAV. Por otro lado, el castellano ha estado en contacto desde antiguo con la lengua vasca, intensificado en los dos últimos siglos por los cambios sociopolíticos, lo que, junto a otros factores, ha influido en este contacto. Por ejemplo, para el castellano en contacto de la CAV se ha notado cierta desdialectalización, en primer lugar, por la presión de la norma, sobre todo a partir de la enseñanza obligatoria y la lengua escrita y, en segundo lugar, por los medios y redes de comunicación ${ }^{10}$. No obstante, es posible que las actitudes y percepciones de los hablantes hacia este contacto, sus resultados y su especificidad, estén ejerciendo una influencia que reclama nuestra atención.

\section{Aproximación a las actitudes lingüísticas de los hablantes}

Desde hace varias décadas, los investigadores están insistiendo en la importancia de las actitudes de los hablantes ante la variación lingüística ${ }^{11}$. Actualmente, se sabe que las elecciones de los hablantes configuran las impresiones de los demás sobre ellos, lo que repercute en los procesos de toma de decisiones en ámbitos tanto sociales como individuales. De hecho, dada la importancia de los procesos psicológicos y sociales para la explicación de ciertos fenómenos sociolingüísticos, las actitudes se han convertido en un componente clave de la construcción de la teoría sociolingüística ${ }^{12}$. En la situación de contacto vasco-castellano, las actitudes de los hablantes respecto a la lengua minoritaria están siendo bastante exploradas ${ }^{13}$. Recientemente se ha observado que los hablantes con actitudes más positivas hacia el euskara son más propensos a incorporar rasgos entonativos de esta lengua en su discurso en castellano ${ }^{14}$. Menos conocidas resultan, en cambio, las actitudes hacia la variedad de castellano de esta zona. Algunos trabajos han advertido que a ciertas variantes lingüísticas del castellano en contacto con la lengua vasca se le atribuyen significados identitarios. Así se ha observado en hablantes urbanos, que consideran expresión de su identidad vasca los préstamos vascos y la articulación particularmente intensa de la vibrante en posición implosiva ${ }^{15}$.

10 La última encuesta sociolingüística sobre las actitudes de la ciudadanía de más de 16 años en relación con la promoción del uso del euskera indica que la posición a favor de la misma es mayoritaria (65\%), en contraste con la posición contraria $(9,3 \%)$ o las actitudes "ni a favor ni en contra" $(25,8 \%)^{16}$. Esta postura ante el fomento de la lengua vasca está estrechamente relacionada con la competencia de esta lengua. A favor de la política lingüística de revitalización del euskera se manifiesta el $87,1 \%$ de los bilingües, el $63,4 \%$ de los bilingües pasivos y el $49,7 \%$ de los castellanohablantes monolingües ${ }^{17}$. 
11 En este último grupo de hablantes, y en el ámbito de la CAV, se han analizado con más detalle las actitudes y prejuicios hacia la lengua vasca ${ }^{18}$. Por ejemplo, en relación con su valor en la construcción del carácter colectivo, para el 46,8\% el euskera es la lengua por excelencia de los vascos, mientras que el 52,3\% otorga el mismo valor al euskera y al castellano. De nuevo, esta opinión está vinculada al sentimiento identitario, en el que la lengua es un elemento sustancial, que está en constante estado de renegociación con otros indicadores de pertenencia al grupo ${ }^{19}$. El 70,2\% de quienes se sienten más vascos que españoles considera que el euskera es la lengua por excelencia; sin embargo, quienes se sienten tan vascos como españoles o más españoles que vascos afirman mayoritariamente (entre el 61 y el 64\%) que el euskera es una de las lenguas.

Con respecto a las razones para aprender la lengua vasca de este mismo grupo de hablantes (castellanohablantes monolingües), quienes declaran estar aprendiendo $(9,9 \%)$ se refieren sobre todo a motivos de tipo instrumental («ayudar a los niños con los deberes» y «optar a más trabajos»); a causas internas («porque es nuestra lengua»); y a las de tipo integrador («poder comunicarme en euskera con los vascohablantes»). Por su parte, quienes declaran no estar aprendiendo euskera $(91,1 \%)$ aducen sobre todo causas relacionadas con el grado de motivación, como tener otras prioridades $(31,9 \%)$, no tener motivación suficiente $(18,9 \%)$, demasiado esfuerzo $(9,7 \%)$ o falta de interés $(6,7 \%)$.

13 Con todo, como es frecuente para los nuevos hablantes de lenguas minoritarias, se ha demostrado que, incluso con un alto grado de competencia (nivel C1 del Marco Común Europeo de Referencia), no todos los neohablantes se sienten plenamente legítimos al hablar euskera ${ }^{20}$. Para algunos existe una diferencia entre saber hablar en euskera y ser un hablante nativo de lengua vasca (un euskaldun), lo que responde a la ideología del hablante nativo como representante legítimo de la identidad lingüística vasca. No obstante, otros neohablantes reivindican la identidad lingüística vasca por el uso habitual del euskera y la adquisición de cierto dominio de formas vernáculas.

\section{La comunicación en escenarios de contacto}

\subsection{Convergencia lingüística}

En escenarios de contacto de lenguas, la convergencia es, sin duda, uno de los mecanismos explicativos de la variación y la innovación lingüística surgida como resultado de ese contacto ${ }^{21}$. Entendido en sentido lato, la convergencia supone el aumento de las similitudes entre dos lenguas en cualquier nivel lingüístico ${ }^{22}$. En este trabajo se interpreta en referencia a la replicación de patrones, es decir, cambios en una forma o estructura de la lengua meta, impulsados por formas o estructuras de la lengua fuente, lo que involucra innovaciones en el significado y posiblemente también en la distribución de estos patrones ${ }^{23}$.

El inicio de los cambios por contacto parte de las necesidades comunicativas de los bilingües, que, comparando patrones o estructuras de las lenguas que conocen, buscan expresiones similares y adecuadas para resolver sus exigencias comunicativas ${ }^{24}$. En esta comparación, el efecto de similitud es fundamental: incluso entre lenguas con grandes diferencias tipológicas, los bilingües detectan similitudes aparentes o superficiales que consideran suficientes para crear estructuras nuevas en la interacción comunicativa ${ }^{25}$. De este modo, los bilingües aprovechan el potencial expresivo de su repertorio 
lingüístico: una estructura o patrón de la lengua fuente desencadena una innovación en función de las tendencias internas de la lengua meta.

Además, está comprobado que las diferencias tipológicas entre las lenguas no impiden cierto grado de confluencia gramatical entre ellas. En español, por ejemplo, son muchos los cambios descritos por convergencia estructural entre esta lengua y lenguas como las amerindias o el inglés ${ }^{26}$. En cuanto al español en contacto con la lengua vasca, este marco está resultando especialmente revelador en zonas de la gramática del español de cierta inestabilidad por su variación interna, así como en marcadores discursivos y adverbios, en los que la ampliación de nuevos significados pragmáticos no requiere procesos de reestructuración. Entre los primeros fenómenos, cabe citar el leísmo con referentes femeninos, la omisión del clítico de objeto directo definido ${ }^{27}$ y cuestiones relativas al orden de palabras ${ }^{28}$; con respecto a los segundos, se encuentra ya como foco de polaridad positiva oracional ${ }^{29} \mathrm{o}$ los valores discursivos de distancia y atenuación de las partículas pospuestas y así y o asi ${ }^{\text {ij }}$.

17 Con respecto a la lengua vasca en contacto con el español, el enfoque de la convergencia explica el marcado diferencial de objeto (DOM) o sobremarcación del dativo ${ }^{31} \mathrm{y}$ ciertos cambios en el orden de palabras ${ }^{32}$. Recientemente, se ha demostrado que las actitudes y creencias de los hablantes en relación con el DOM influyen en su uso. Como se trata de un fenómeno estigmatizado en la lengua estándar, marcado como rasgo de identidad vasca "menos auténtica", es más frecuente en bilingües nativos de lengua vasca que en aprendientes avanzados de vasco como segunda lengua, lo que revalida la importancia de la conciencia metalingüística en los usos y preferencias lingüísticas de los hablantes ${ }^{33}$.

18 A modo de ilustración del mecanismo de la convergencia entre el español y la lengua vasca, tratamos brevemente el orden de palabras. Aunque las dos lenguas coinciden en la relativa flexibilidad en el ordenamiento de constituyentes, el esquema neutro del euskera es SOV con posición del foco preverbal (1a), frente al SVO del español con foco posverbal (1b). En una interacción neutra, a la pregunta de a quién vio Jon, los constituyentes se ordenarían así:

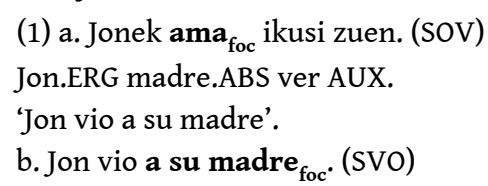

19 La alteración del orden neutro en ambas lenguas se explica por razones pragmáticoinformativas. Así, en español, el orden OV se usa para el mantenimiento del tema o para la introducción de temas nuevos. Sin embargo, en el español en contacto se ha detectado, además de un porcentaje mayor de OV (2), una mayor frecuencia de OV con información nueva, que no se utiliza para la introducción de un tema nuevo, porque el referente de este objeto preverbal no se mantiene en el discurso subsecuente ${ }^{34}$. Por lo tanto, en los bilingües ${ }^{35}$ ha aumentado la frecuencia de un orden posible en el castellano (OV), pero con las características informativas de la lengua vasca.

(2) Otra cosa tenía pensa[d]o, pero ahora no me acuerdo qué quería explicar ${ }^{36}$.

En lo que se refiere a la lengua vasca, se ha advertido un uso creciente de ordenamientos de constituyentes que, si bien en ciertos contextos son posibles para esta lengua, resultan muy próximos al orden del español, como en (3), donde se reproduce una oración en lengua vasca con un orden muy ajustado al español. 
(3) Ahal doguz repetidu danak? ${ }^{37}$.

poder AUX repetir todos.ABS

¿¿Podemos repetirlos todos?'

21 En efecto, en vasco estándar el grupo verbal se ordenaría repetidu ahal doguz ('podemos repetirlo'), en contraste con el orden del español poder repetir; igualmente, el cuantificador danak ('todos') aparece en posición de foco posverbal, como en español, en lugar de la posición preverbal propia de la lengua vasca (Danak repetidu ahal doguz). En definitiva, tanto en (2) como en (3) las innovaciones están condicionadas por la posición del foco de la lengua en contacto y se desarrollan dentro de las posibilidades de ordenamiento de cada una de las lenguas meta.

\subsection{Cambio de código o code-switching}

El cambio de código (code-switching), es decir, la capacidad de los bilingües de usar sus lenguas dentro de un mismo acto comunicativo, está siendo estudiado desde comienzos de la década de los 70 del siglo pasado ${ }^{38}$. La tipología de los cambios de código se asienta sobre los criterios proporcionados tanto por la perspectiva gramatical como por la comunicativa. Así, desde el punto de vista sintáctico se distinguen (a) los cambios que ocurren en unidades periféricas del discurso (enlaces extraoracionales, marcadores discursivos, interjecciones, unidades parentéticas, coletillas); (b) los cambios interoracionales, que afectan a enunciados oracionales a partir de un conector o una pausa; y (c) los cambios de código intraoracionales, que suceden en el interior de la oración. A esta tipología sintáctica se han superpuesto otras de tipo pragmático y discursivo, como el hecho de que el cambio de código ocurra entre actos de habla diferentes o dentro de un mismo acto de habla, o responda a una estrategia del discurso.

En la situación de contacto que se describe en estas páginas, el cambio de código conversacional se ha analizado como recurso comunicativo adicional de los bilingües desde los 90 del siglo pasado ${ }^{39}$. Además de caracterizarse como una práctica frecuente y natural, que requiere menor esfuerzo que el mantenimiento en una sola lengua ${ }^{40}$, se han descrito algunos aspectos como el cambio interoracional, que responde al patrón "euskaraz hasten dut ('empiezo en euskara') y termino en español" ${ }^{41}$, y los factores que guían la asignación del género en el marco de las construcciones nominales ${ }^{42}$. En cuanto a sus funciones, se extienden desde la necesidad de llenar vacíos léxicos hasta la organización del discurso, pasando por estrategias de refuerzo y atenuación, la introducción de nuevos temas y la regulación de los turnos de habla ${ }^{43}$.

A modo de ilustración se presentan los ejemplos de (4), cambios de código que responden a estrategias de refuerzo y énfasis sobre el tiempo que el hablante lleva sin celebrar su cumpleaños (4a); por su parte, en (4b) la expresión horas muertas puede colmar un vacío fraseológico en lengua vasca (horas muertas, 'horas que se pasan sin hacer nada o en una ocupación sin provecho', según DRAE, s.v. hora); para (4c) el cambio de código resalta una cita, lo que de algún modo refuerza el valor de verdad de lo enunciado; en (4d) la secuencia cena del Regional responde a una estrategia de refuerzo y precisión de lo que se transmite. No obstante, conviene recordar que no todos los cambios de lengua orales albergan un propósito comunicativo particular, sino que constituyen otra manera de hablar de los bilingües, en este caso, jóvenes de Pamplona (Navarra) entre 18 y 25 años ${ }^{44}$. 
(4) a. Nire urtebetetzerako ('para mi cumpleaños'), que ez dut ospatzen ('no lo he celebrado') desde hace 3 años.

b. Ta hantxe egon ginen ('y ahí estuvimos') horas muertas.

c. Esan nion ('le dije') que no me parecía normal tampoco.

d. Titok dauka ('Tito tiene') cena del Regional, seguro joango dela ('que irá').

Más allá de las funciones pragmáticas y retóricas, Muysken ${ }^{45}$ alude a ciertos aspectos que favorecen el cambio de código por parte de los bilingües. Entre ellos, destaca la considerable distancia tipológica y léxica entre las dos lenguas; su situación de cierta tensión en el ámbito político; así como el grado de dominio del hablante de las lenguas implicadas. De hecho, el cambio de código no puede interpretarse como indicio de falta de competencia del bilingüe o de incapacidad para separar las lenguas. Se trata, más bien, de una manifestación de su destreza con ambos repertorios, pues quienes lo practican son hablantes capaces de expresarse con suficiente fluidez en las dos lenguas ${ }^{46}$.

En relación con lo anterior y ligado a las actitudes, el cambio de código puede servir como marcador de solidaridad y pertenencia a un grupo. Con todo, los bilingües ${ }^{47}$ lo interpretan como propio de contextos informales y lo valoran de forma diferente según el origen lingüístico del hablante: si este es nativo de lengua vasca, el cambio de código se considera natural, mientras que se considera una falta de competencia para quienes el vasco es su segunda lengua.

\section{Recapitulación}

En este trabajo se ha sintetizado el contexto sociolingüístico del contacto vascocastellano, así como a las actitudes de los hablantes bilingües y monolingües en castellano hacia la lengua vasca. En el marco del contexto sociolingüístico referido, se han explicado dos de los varios resultados del contacto desde el punto de vista conversacional y lingüístico. Por una parte, se ha presentado el concepto de convergencia lingüística y su papel en la explicación de los cambios inducidos por contacto en las dos lenguas; por otra parte, se ha tratado el cambio de código conversacional.

Para ambos fenómenos se ha destacado el peso tanto de las características de las lenguas implicadas como de los factores sociales. Con respecto a la convergencia, se ha mostrado que los cambios desencadenados por la lengua fuente se suelen acomodar en la lengua meta según sus tendencias internas. No obstante, las ideologías y actitudes lingüísticas influyen en los usos de los hablantes, como sucede con el DOM en lengua vasca: resulta más habitual en bilingües nativos que en aprendientes avanzados de euskera por su valoración como rasgo de identidad vasca "menos auténtica". En cuanto al cambio de código, es un fenómeno favorecido por la notable distancia tipológica y léxica entre vasco y castellano. Pero, pese a su importancia como marcador identitario, de nuevo se valora negativamente para quienes el vasco es su segunda lengua, mientras que se considera natural para los bilingües nativos.

Los aspectos presentados en este trabajo vuelven a poner de manifiesto la necesaria multidisciplinariedad del análisis de los escenarios de contacto. En concreto, en lo que respecta al contacto vasco-español, es urgente la investigación sobre percepción, creencias y actitudes tanto en lo que respecta a la variedad de castellano de contacto, como en relación con otros fenómenos como el cambio de código o el préstamo 
lingüístico. Investigando los factores actitudinales cognitivos y afectivos que subyacen a la variación y al cambio inducidos por contacto lingüístico podremos comprender mejor el comportamiento de los hablantes respecto a sus lenguas.

\section{BIBLIOGRAFÍA}

Estibaliz AMORRORTU, Ana ORTEGA, Itziar IDIAZABAL y Andoni BARREÑA, Actitudes y prejuicios de los castellanohablantes hacia el euskera, Vitoria-Gasteiz, Servicio de Publicaciones del Gobierno Vasco, 2009.

Gotzon AURREKOETXEA y Lorea UNAMUNO, «Perpaus arteko euskara-gaztelania kodealdaketa», Euskalingua, no 19 (2011), p. 25-30.

Maria-Jose AZURMENDI, Nekane LARRAÑAGA y Jokin APALATEGI, «Bilingualism, identity, and citizenship in the Basque Country», Mercedes Niño-Murcia y Jason Rothman, Bilingualism and identity: Spanish at the crossroads with other languages, Amsterdam, John Benjamins, 2009, p. 35-86.

Patxi BAZTARRIKA GALPARSORO, «El euskera: un caso de revitalización», Javier Giralt Latorre y Francho Nagore Laín (eds.), La normalización social de las lenguas minoritarias, Zaragoza, Prensas de la Universidad de Zaragoza, 2019, p. 83-146.

Kurt BRAUNMÜLLER, Steffen HÖDER, Karoline KÜHL (eds.), Stability and Divergence in Language Contact: Factors and Mechanisms, Amsterdam, John Benjamins, 2014.

Barbara E. BULLOCK y A. Jacqueline TORIBIO, «Themes in the study of code-switching», Barbara E. Bullock y A. Jacqueline Toribio (eds.), The Cambridge Handbook of Linguistic Code-switching, Cambridge, Cambridge University Press, 2009, p. 1-17.

Bruno CAMUS y Sara GómEZ SEIBANE, «Introducción», Bruno Camus y Sara Gómez Seibane (eds.), El castellano del País Vasco, Vitoria-Gasteiz, UPV-EHU, 2012, p. 1-17.

Bruno CAMUS y Sara GómEZ SEIBANE, «Nuevos datos acerca de la omisión de objetos en el castellano del País Vasco», Círculo de Lingüística Aplicada a la Comunicación, oㅡ 61 (2015), p. 211-236.

Bruno CAMUS y Sara GómEZ SEIBANE, «Modalización por contacto en el castellano del País Vasco», RILI, nº 32/2 (2018), p. 21-33.

$\mathrm{M}^{\mathrm{a}}$ del Puy CIRIZA, Dialect divergence and identity in Basque Spanish, Tesis doctoral, University of Illinois at Urbana-Champaign, 2009.

Angela DE BRUIN, Arthur G. SAMUEL y Jon A. DUÑABEITIA, «Voluntary language switching: When and why do bilinguals switch between their languages?», Journal of Memory and Language, $\mathrm{n}^{\circ} 103$ (2018), p. 28-43.

Gorka ELORDIETA y Magdalena ROMERA, «The influence of social factors on the prosody of Spanish in contact with Basque», International Journal of Bilingualism, nº 25/1 (2020) <DOI:

$10.1177 / 1367006920952867>$.

Inés FERNÁNDEZ-ORDóÑEZ, «Leísmo, laísmo y loísmo», Ignacio Bosque y Violeta Demonte (dirs.), Gramática descriptiva de la lengua española, Madrid, Espasa-Calpe, vol. 1, 1999, p. 1317-1397. 
Peter GARRETT, «Language attitudes and sociolinguistics», Journal of Sociolinguistics, ํo 5 (2001), p. 626-631.

Howard GILES y Andrew C. BILLINGS, «Assessing Language Attitudes: Speaker Evaluation Studies», Alan Davies y Catherine Elder (eds.), Handbook of Applied Linguistics, Oxford, Blackwell, 2004, p. 187-209.

GOBIERNO De nAVARRA, Datos sociolingüísticos de Navarra, Pamplona, Servicio de Publicaciones, 2020 GOBIERno VASCo, V Mapa Sociolingüístico, Vitoria-Gasteiz, Servicio Central de Publicaciones, 2014. GOBIERNO VASCO y GOBIERNo De NAVARRA, VI Encuesta Sociolingüística. Comunidad Autónoma de Euskadi, 2016.

Sara GÓMEZ SEIBANE, «El peso de la norma lingüística en la configuración del castellano del País Vasco: una mirada al siglo XIX», Eugenio Bustos Gisbert y Juan Sánchez Méndez (eds.), La configuración histórica de las normas del castellano, Valencia, Tirant lo Blanch, 2019, p. 249-267.

Sara GÓMEZ SEIBANE y José L. RAMÍREZ LUENGO, «Variación morfosintáctica y aceptabilidad normativa en el español del País Vasco: una aproximación», Español Actual, nº 101 (2014), p. 31- 45.

Sara GómEZ SEIBANE, «Contacto de lenguas y orden de palabras: OV/VO en el español del País Vasco», Lingüística Española Actual, nº 34/1 (2012), p. 115-135.

Sara GÓMEZ SEIBANE, «Variación interdialectal de o así: de la atenuación de lo dicho a la atenuación del decir», Lingüística Española Actual, no 41/1 (2018), p. 87-110.

Sara GÓMEZ SEIBANE «Gramaticalización, modalización y contacto: y así en dos variedades de español», Círculo de Lingüística Aplicada a la Comunicación, ํำ 75 (2018), p. 137-154.

Sara GÓMEZ SEIBANE, «El español en contacto con la lengua vasca: orden de palabras y estructura informativa en diacronía», Marta López Izquierdo y Mónica Castillo Lluch (eds.), El orden de palabras en la historia del español, Madrid, Visor, 2015, p. 457-482.

Orreaga IBARRA, «Tipología y pragmática del code-switching vasco-castellano en el habla informal de jóvenes bilingües», Lapurdum, nº 18 (2014), p. 23-40.

Alazne LANDA, Conditions on Null Objects in Basque Spanish and their relation to Leísmo and Clitic Doubling, PhD Dissertation, University of Southern California, 1995.

Hanna LANTTO, «Conversations about code-switching: contrasting ideologies of purity and authenticity in Basque bilinguals’ reactions to bilingual speech», Multilingua, no 35/2 (2016), 137-161.

David LASAGABASTER, «Language use and language attitudes in the Basque Country», David Lasagabaster y Angel Huguet (eds.), Multilingualism in European Bilingual Contexts. Language Use and Attitudes, Clevedon, Multilingual Matters, 2007, p. 65-89.

Yaron MATRAS, Language Contact, Cambridge, Cambridge University Press, 2009.

Inma MUÑOA, «Pragmatic functions of code-switching among Basque-Spanish bilinguals», Actas do I Simposio Internacional sobre o Bilingüismo, Vigo, Universidade de Vigo, 1997, p. 528-541.

Silvina MONTRUL, Incomplete Acquisition in Bilingualism. Re-examining the Age Factor, Amsterdam, John Benjamins, 2009.

Pieter MUYSKEN, «Language contact outcomes as the result of bilingual optimization strategies», Bilingualism: Language and Cognition, $\mathrm{n}^{\circ}$ 16/4 (2013), p. 709-730. 
Mercedes NIÑO-MURCIA y Jason ROTHMAN, «Spanish-contact bilingualism and identity», Bilingualism and identity: Spanish at the crossroads with other languages, Amsterdam, John Benjamins, 2009, p. 12.32 .

Ane ORTEGA, Jacqueline URLA, Estibaliz AMORRORTU, Jone GOIRIGOLZARRI y Belen URANGA, «Linguistic identity among new speakers of Basque», International Journal of the Sociology of Language, $\mathrm{n}^{\circ} 231$ (2015), p. 85-105.

Jon ORTIZ DE URBINA, «Word Order», Ane Berro, Beatriz Fernández y Jon Ortiz de Urbina (eds), Basque and Romance. Aligning Grammars, Leiden / Boston, Brill, 2019, p. 14-58.

Azucena PALACIOS, «Contact-induced change and internal evolution: Spanish in contact with Amerindian languages», Claudine Chamoreau y Isabelle Léglise (eds.), The Interplay of Variation and Change in Contact Settings, Amsterdam / Philadelphia, John Benjamins, 2013, p. 165-198 Azucena PALACIOS y Stephan PFÄNDER, «Similarity effects in language contact: Taking the speakers' perceptions of congruence seriously», Juliane Besters-Dilger, Cynthia Dermarkar, Stephan Pfänder \& Achim Rabus (eds.), Congruence in Contact-Induced Language Change. Language Families, Typological Resemblance, and Perceived Similarity, Berlin/Boston, De Gruyter, 2014, p. 219-238.

M Carmen PARAFITA COUTO, Amaia MUNARRIZ, Irantzu EPELDE, Margaret DEUCHAR y Bernard OYHARÇABAL, «Gender conflict resolution in Spanish - Basque mixed DPs», Bilingualism: Language and Cognition, $\mathrm{n}^{\circ}$ 18/2 (2015), p. 304-323.

Shana POPLACK, «Sometimes I'll Start a Sentence in Spanish y termino en español: Toward a Typology of Code-Switching», Linguistics, no 18 (1980), p. 581-618

ItXaso RODRÍGUEZ-ORDÓÑEZ, «The Role of Linguistic Ideologies in Language Contact Situations», Language and Linguistic Compass, nº e12351, 2019, <URL: https://doi.org/10.1111/lnc3.12351>.

ItXaso RODRÍGUEZ-ORDóÑEZ, «Reexamining Differential Object Marking as a Linguistic ContactPhenomenon in Gernika Basque», Journal of Language Contact, $n^{\circ}=10 / 2$ (2017), p. 318-352.

Itxaso RODRÍGUEZ-ORDÓÑEZ, «The acquisition of Differential Object Marking in Basque as a sociolinguistic variable», Alexandru Mardale y Silvina Montrul (eds.), The acquisition of Differential Object Marking, Amsterdam, John Benjamins, 2020, p. 105-131.

Carmen SILVA-CORVALÁN, Language Contact and Change: Spanish in Los Angeles, Oxford, Clarendon Press, 1994.

Carmen SILVA-CORVALÁN, «The limits of convergence in language contact», Journal of Language Contact, no 2/1 (2008), p. 213-224, <URL: https://doi.org/10.1163/000000008792525246>.

Robert L. TRASK, The history of Basque, London / New York, Routledge, 1997, p. 23-25.

Donald WINFORD, «Theories of language contact», Anthony P. Grant (ed.), The Oxford Handbook of Language Contact, New York, Oxford University Press, 2020, p. 51-74.

\section{NOTAS}

1. Pieter MUYSKEN, «Language contact outcomes as the result of bilingual optimization strategies», Bilingualism: Language and Cognition, $\mathrm{n}^{\circ}$ 16/4 (2013), p. 709-730. Donald WINFORD, «Theories of language contact», Anthony P. Grant (ed.), The Oxford Handbook of Language Contact, New York, Oxford University Press, 2020, p. 51-74. 
2. En este trabajo se utilizan como sinónimos castellano y español. No obstante, el primero es el nombre que sus usuarios y hablantes prefieren mayoritariamente en la zona de contacto analizada. Asimismo, son sinónimas lengua vasca y euskera. Vid. Bruno CAMUS y Sara GómEZ SEIBANE, «Introducción», Bruno Camus y Sara Gómez Seibane (eds.), El castellano del País Vasco, VitoriaGasteiz, UPV-EHU, 2012, p. 1-17.

3. En líneas generales, la comunidad científica comparte una idea general de qué significa ser bilingüe y de quién puede considerarse como tal. Según Montrul, el bilingüe posee conocimiento estable de dos o más lenguas (relativamente fijo y no necesariamente nativo), y control funcional de las mismas, sin importar el nivel de conocimiento o de uso en la vida cotidiana. Silvina MONTRUL, Incomplete Acquisition in Bilingualism. Re-examining the Age Factor, Amsterdam, John Benjamins, 2009. Sin embargo, el bilingüismo es un fenómeno complejo desde distintas perspectivas (cognitiva, funcional y social) y depende de variables como el orden de adquisición de cada lengua, el grado de uso, el contexto sociopolítico, el estatus de las lenguas en la sociedad (mayoritaria o minoritaria), la edad y el contexto de adquisición, así como el nivel de conocimiento de las dos lenguas.

4. Sara GÓMEZ SEIBANE, «El peso de la norma lingüística en la configuración del castellano del País Vasco: una mirada al siglo XIX», Eugenio Bustos Gisbert y Juan Sánchez Méndez (eds.), La configuración histórica de las normas del castellano, Valencia, Tirant lo Blanch, 2019, p. 249-267.

5. Robert L. TRASK, The history of Basque, London / New York, Routledge, 1997, p. 23-25.

6. Patxi BAZTARRIKA GALPARSORO, «El euskera: un caso de revitalización», Javier Giralt Latorre y Francho Nagore Laín (eds.), La normalización social de las lenguas minoritarias, Zaragoza, Prensas de la Universidad de Zaragoza, 2019, p. 83-146.

7. GobIeRno VAsco, V Mapa Sociolingüístico, Vitoria-Gasteiz, Servicio Central de Publicaciones, 2014,

p. 29.

8. GOBIERNO VASCO, op. cit, p. 30.

9. Gobierno de navarRA, Datos sociolingüísticos de Navarra, Pamplona, Servicio de Publicaciones, 2020.

10. Sara GÓMEZ SEIBANE y José L. RAMÍREZ LUENGO, «Variación morfosintáctica y aceptabilidad normativa en el español del País Vasco: una aproximación», Español Actual, no 101 (2014), p. 31-45.

11. Howard GILES y Andrew C. BILLINGS, «Assessing Language Attitudes: Speaker Evaluation Studies», Alan Davies y Catherine Elder (Eds.), Handbook of Applied Linguistics, Oxford, Blackwell, p. 187-209, 2004.

12. Peter GARRETT, «Language attitudes and sociolinguistics», Journal of Sociolinguistics, no 5 (2001), p. 626-631.

13. Cabe citar, entre otros, los trabajos de David LASAGABASTER, «Language use and language attitudes in the Basque Country», David Lasagabaster y Angel Huguet (eds.), Multilingualism in European Bilingual Contexts. Language Use and Attitudes, Clevedon, Multilingual Matters, 2007, p. 65-89; Maria-Jose AZURMENDI, Nekane LARRAÑAGA y Jokin APALATEGI, «Bilingualism, identity, and citizenship in the Basque Country», Mercedes Niño-Murcia y Jason Rothman, Bilingualism and identity: Spanish at the crossroads with other languages, Amsterdam, John Benjamins, 2009, 35-86; ItXaso RODRÍGUEZ-ORDÓÑEZ, "The Role of Linguistic Ideologies in Language Contact Situations», Language and Linguistic Compass, no e12351 (2019), <URL: https://doi.org/10.1111/lnc3.12351>.

14. Gorka ELORDIETA y Magdalena ROMERA, «The influence of social factors on the prosody of Spanish in contact with Basque», International Journal of Bilingualism, no 25/1, 2021, <DOI: 10.1177/1367006920952867>.

15. $\mathrm{M}^{\mathrm{a}}$ del Puy CIRIZA, Dialect divergence and identity in Basque Spanish, Tesis doctoral, University of Illinois at Urbana-Champaign, 2009.

16. GOBIERNO VASCO y GOBIERNO DE NAVARRA, VI Encuesta Sociolingüística. Comunidad Autónoma de Euskadi, 2016, p. 27. 
17. Estos perfiles lingüísticos responden a las mismas características que los del apartado 2 . Recordamos aquí que bilingüe es quien habla bien o bastante bien en castellano y lengua vasca, bilingüe pasivo es quien conoce y usa el castellano, pero en lengua vasca solo tiene competencias receptivas, y como monolingüe se categorizan los que solo saben y usan el castellano. GOBIERNO VASCO Y GOBIERNO DE NAVARRA, op. cit.

18. Estibaliz AMORRORTU, Ana ORTEGA, Itziar IDIAZABAL y Andoni BARREÑA, Actitudes y prejuicios de los castellanohablantes hacia el euskera, Vitoria-Gasteiz, Servicio de Publicaciones del Gobierno Vasco, 2009. El perfil sociolingüístico de estos hablantes es el mismo que el de la nota anterior.

19. Mercedes NIÑO-MURCIA y Jason ROTHMAN, "Spanish-contact bilingualism and identity», Bilingualism and identity: Spanish at the crossroads with other languages, Amsterdam, John Benjamins, 2009, p. 12-32.

20. Ane ORTEGA, Jacqueline URLA, Estibaliz AMORRORTU, Jone GOIRIGOlZARRI y Belen URANGA, «Linguistic identity among new speakers of Basque», International Journal of the Sociology of Language, 231 (2015), 85-105.

21. Kurt BRAUNMÜLLER, Steffen HÖDER, Karoline KÜHL (eds.), Stability and Divergence in Language Contact: Factors and Mechanisms, Amsterdam, John Benjamins, 2014.

22. Carmen SILVA-CoRvalán, Language Contact and Change: Spanish in Los Angeles, Oxford, Clarendon Press, 1994, p. 4-5.

23. Yaron MATRAS, Language Contact, Cambridge, Cambridge University Press, 2009, p. 238.

24. Yaron MATRAS, op. cit.

25. Azucena PALACIOS y Stephan PFÄNDER, «Similarity effects in language contact: Taking the speakers' perceptions of congruence seriously», Juliane Besters-Dilger, Cynthia Dermarkar, Stephan Pfänder y Achim Rabus (eds.), Congruence in Contact-Induced Language Change. Language Families, Typological Resemblance, and Perceived Similarity, Berlin/Boston, De Gruyter, 2014, p. 219-238.

26. Entre otros, Azucena PALACIOS, «Contact-induced change and internal evolution: Spanish in contact with Amerindian languages», Claudine Chamoreau y Isabelle Léglise (eds.), The Interplay of Variation and Change in Contact Settings, Amsterdam / Philadelphia, John Benjamins, 2013, p. 165-198. Carmen SILVA-CORVALÁN, "The limits of convergence in language contact», Journal of Language Contact, no 2/1 (2008), p. 213-224, <URL: https://doi.org/10.1163/000000008792525246>.

27. Alazne LANDA, Conditions on Null Objects in Basque Spanish and their relation to Leísmo and Clitic Doubling, PhD Dissertation, University of Southern California, 1995. Inés FERNÁNDEZ-ORDóÑEZ, «Leísmo, laísmo y loísmo», Ignacio Bosque y Violeta Demonte (dirs.), Gramática descriptiva de la lengua española, Madrid, Espasa-Calpe, vol. 1, 1999, p. 1317-1397. Bruno CAMUS y Sara GómEZ SEIBANE, «Nuevos datos acerca de la omisión de objetos en el castellano del País Vasco», Círculo de Lingüística Aplicada a la Comunicación, no 61 (2015), p. 211-236.

28. Sara GómeZ SEIBANE, «Contacto de lenguas y orden de palabras: OV/VO en el español del País Vasco», Lingüística Española Actual, no 34/1 (2012), p. 115-135.

29. Bruno CAMUS y Sara GómEZ SEIBANE, «Modalización por contacto en el castellano del País Vasco», RILI, no 32/2 (2018), p. 21-33.

30. Sara GÓMEZ SEIBANE, "Variación interdialectal de o así: de la atenuación de lo dicho a la atenuación del decir», Lingüística Española Actual, nº 41/1 (2018), p. 87-110. Sara GóMEZ SEIBANE «Gramaticalización, modalización y contacto: $y$ así en dos variedades de español», Círculo de Lingüística Aplicada a la Comunicación, no 75 (2018), p. 137-154.

31. Itxaso RODRÍGUEZ-ORDÓÑEZ, «Reexamining Differential object Marking as a Linguistic ContactPhenomenon in Gernika Basque», Journal of Language Contact, no 10/2 (2017), p. 318-352.

32. Jon ORTIZ DE URBINA, «Word Order», Ane Berro, Beatriz Fernández y Jon Ortiz de Urbina (eds.), Basque and Romance. Aligning Grammars, Leiden / Boston, Brill, 2019, p. 14-58. 
33. Itxaso RODRÍGUEZ-ORDóÑEZ, «The acquisition of Differential Object Marking in Basque as a sociolinguistic variable», Alexandru Mardale y Silvina Montrul (eds.), The acquisition of Differential Object Marking, Amsterdam, John Benjamins, 2020, p. 105-131.

34. Sara GÓMEZ SEIBANE, op. cit., 2012. Sara GÓMEZ SEIBANE, «El español en contacto con la lengua vasca: orden de palabras y estructura informativa en diacronía», Mónica Castillo Lluch y Marta López Izquierdo (eds.), El orden de palabras en la historia del español, Madrid, Visor, 2015, p. 457-482. 35. En Sara GÓMEZ SEIBANE, op. cit., 2012, p. 117-118, se utilizan datos de 2010 de distintos perfiles de bilingües: hablantes entre 25 y 50 años con habilidades productivas de expresión oral y escrita en ambas lenguas; bilingües de la misma edad con competencia pasiva en lengua vasca, con español como lengua primera y dominante; y bilingües mayores de 60 años con preponderancia de la lengua vasca y un nivel de instrucción bajo.

36. Sara GómEZ SEIBANE, op. cit., 2012, p. 20.

37. Jon ORTIZ DE URBINA, op. cit., p. 54. Se trata de un ejemplo extraído de un joven bilingüe con la variedad vizcaína de euskara como lengua nativa.

38. Barbara E. BULLOCK y A. Jacqueline TORIBIO, «Themes in the study of code-switching», Barbara

E. Bullock y A. Jacqueline Toribio (eds.), The Cambridge Handbook of Linguistic Code-switching, Cambridge, Cambridge University Press, 2009, p. 1-17.

39. Hanna LANTTO, «Conversations about code-switching: contrasting ideologies of purity and authenticity in Basque bilinguals' reactions to bilingual speech», Multilingua, $\mathrm{n}^{\mathrm{o}}$ 35/2 (2016), 137-161.

40. Angela DE BRUIN, Arthur G. SAMUEL y Jon A. DUÑABEITIA, «Voluntary language switching: When and why do bilinguals switch between their languages?», Journal of Memory and Language, nㅡ 103 (2018), p. 28-43. Los experimentos se realizaron con bilingües entre 18 y 35 años (p. 30 y 33), que habían completado su educación primaria y secundaria en euskara, por lo que tienen un alto grado de competencia en las dos lenguas, adquiridas antes de los 7 años.

41. Gotzon AURREKOETXEA y Lorea UNAMUNO, «Perpaus arteko euskara-gaztelania kodealdaketa», Euskalingua, no 19 (2011), p. 25-30. Los datos para el análisis se tomaron de bilingües con euskara como lengua nativa y dominante.

42. $M^{\mathrm{a}}$ Carmen PARAFITA Couto, Amaia MUNARRIZ, Irantzu EPELDE, Margaret DeUChAR y Bernard OYHARÇABAL, «Gender conflict resolution in Spanish - Basque mixed DPs», Bilingualism: Language and Cognition, no 18/2 (2015), p. 304-323. Los datos se tomaron de 30 bilingües entre 20 y 30 años con altas competencias en ambas lenguas; de ellos, 26 tenían el castellano como primera lengua y adquirieron el euskara en la escuela por inmersión lingüística; los 4 restantes eran bilingües nativos.

43. Inma MUÑOA, «Pragmatic functions of code-switching among Basque-Spanish bilinguals», Actas do I Simposio Internacional sobre o Bilingüismo, Vigo, Universidade de Vigo, 1997, p. 528-541.

44. Orreaga IBARRA, «Tipología y pragmática del code-switching vasco-castellano en el habla informal de jóvenes bilingües», Lapurdum, no 18 (2014), p. 23-40. Vid. p. 29, 32, 33, 36. Son bilingües para quienes el euskara es su lengua de estudio desde los tres años, por lo que poseen una competencia alta en lengua escrita y oral; en la vida familiar y en el hogar, sin embargo, predomina el uso del castellano.

45. Pieter MUYSKEN, op. cit., p. 714-716.

46. Shana POPLACK, «Sometimes I'll Start a Sentence in Spanish y termino en español: Toward a Typology of Code-Switching», Linguistics, no 18 (1980), p. 581-618. Pieter MUYSKEN, op. cit., p. 713-714.

47. Hanna LANTTO, op. cit., p. 144. En este caso, se trata de bilingües de entre 19 y 57 años, la mitad de los cuales tenían como lengua nativa el euskara, de diversos grupos profesionales. Los datos se grabaron entre diciembre de 2011 y enero de 2012 en Bilbao y Barakaldo (Bizkaia). 


\section{RESÚMENES}

L'article expose le contexte sociolinguistique du contact basque-espagnol, avec une attention particulière aux politiques linguistiques du XXe siècle, ainsi qu'aux attitudes des hispanophones bilingues et monolingues à l'égard de la langue basque. Dans ce contexte, deux résultats du contact sont présentés d'un point de vue conversationnel et linguistique. D'une part, la convergence linguistique et son rôle dans l'explication des changements provoqués par le contact dans les deux langues; d'autre part, l'alternance codique au sein d'une conversation. Pour les deux phénomènes, il ressort l'importance des caractéristiques structurelles des langues impliquées ainsi que des facteurs sociolinguistiques. Cela met en évidence la nécessité d'une approche multidisciplinaire pour l'analyse du contact linguistique.

This paper summarises the sociolinguistic context of Basque-Spanish contact, with special attention to the language policies of the 20th century, as well as the attitudes of bilingual and monolingual speakers in Spanish towards the Basque language. In this framework, two outcomes of language contact are explained from a conversational point of view. On one hand, linguistic convergence and its role in explaining the contact induced changes in the two languages; on the other hand, the code-switching in the context of a single conversation. For both phenomena, languages involved structural characteristics and sociolinguistic factors are essential to our understanding of language contact outcomes. This highlights the multidisciplinary nature of the analysis of contact linguistics.

\section{ÍNDICE}

Keywords: Basque-Spanish contact, linguistic convergence, code-switching, speaker's attitudes Mots-clés: contact basque-espagnol, convergence linguistique, changement de code, attitudes de l'orateur

\section{AUTOR}

\section{SARA GÓMEZ SEIBANE}

Universidad de La Rioja 